\title{
Contrast-enhanced ultrasound imaging can be used to evaluate fetal cerebral perfusion: potential implications for fetal surgery
}

Kendall M. Lawrence ${ }^{1}$, Barbara E. Coons ${ }^{1}$, Anush Sridharan², Avery C. Rossidis ${ }^{1}$, Marcus G. Davey', Alan W. Flake ${ }^{1,3}$ and Ryne A. Didier ${ }^{2,3^{*}}$

\begin{abstract}
Background: Fetal surgery is increasingly performed to correct congenital defects. Currently, fetal brain perfusion cannot be assessed intra-operatively. The purpose of this study was to determine if contrast-enhanced ultrasound (CEUS) could be used to monitor fetal cerebral perfusion during fetal surgery and if parameters correlate with fetal hemodynamics or acid/base status.

Methods: Cannulated fetal sheep were insufflated with carbon dioxide gas in an extra-uterine support device and in utero to mimic fetal surgery. Fetal heart rate, mean arterial pressure, and arterial blood gases were serially measured. CEUS examinations of the brain were performed and time-dependent metrics were quantified to evaluate perfusion. The relationships between measured parameters were determined with mixed linear effects models or two-way repeated measures analysis of variance.

Results: 6 fetal sheep (113 \pm 5 days) insufflated at multiple time-points ( $n=20$ experiments) in an extra-uterine support device demonstrated significant correlations between time-dependent perfusion parameters and fetal $\mathrm{pH}$ and carbon dioxide levels. In utero, 4 insufflated fetuses (105 \pm 1 days) developed hypercarbic acidosis and had reductions in cerebral perfusion parameters compared to age-matched controls $(n=3)$. There was no significant relationship between cerebral perfusion parameters and fetal hemodynamics.
\end{abstract}

Conclusions: CEUS-derived cerebral perfusion parameters can be measured during simulated fetal surgery and strongly correlate with fetal acid/base status.

Keywords: Ultrasonography, Microbubbles, brain, Contrast media, Models, animal, Fetal surgery, Brain perfusion

\footnotetext{
*Correspondence: didierr@email.chop.edu

2Department of Radiology, The Children's Hospital of Philadelphia, 3401 Civic

Center Blvd., PA PA 19104 Philadelphia, USA

${ }^{3}$ Perelman School of Medicine, University of Pennsylvania, Pennsylvania,

Philadelphia, USA

Full list of author information is available at the end of the article
}

(c) The Author(s). 2021 Open Access This article is licensed under a Creative Commons Attribution 4.0 International License, which permits use, sharing, adaptation, distribution and reproduction in any medium or format, as long as you give appropriate credit to the original author(s) and the source, provide a link to the Creative Commons licence, and indicate if changes were made. The images or other third party material in this article are included in the article's Creative Commons licence, unless indicated otherwise in a credit line to the material. If material is not included in the article's Creative Commons licence and your intended use is not permitted by statutory regulation or exceeds the permitted use, you will need to obtain permission directly from the copyright holder. To view a copy of this licence, visit http://creativecommons.org/licenses/by/4.0/. 


\section{Background}

Fetal surgery is being increasingly utilized to correct severe congenital defects in utero prior to birth. Over the past three decades, the field of fetal surgery has advanced rapidly with increasingly complex intrauterine procedures now performed at more than fifty fetal centers in the United States $[1,2]$. These surgeries are performed at mid-gestation via maternal laparotomy and hysterotomy, or more recently, via hysterotomy-sparing endoscopy with partial amniotic carbon dioxide insufflation (PACI) to aid visualization [3-5]. During these complex surgeries, maternal hemodynamics, blood gases and lactate levels are continuously monitored via arterial access, but analogous parameters cannot be monitored in the fetus without invasive procedures such as arterial line placement and repeated serum blood gas analysis, which are prohibitively dangerous at mid-gestation [6].

As fetal surgeries continue to become more complex, there is concern that prolonged anesthesia and carbon dioxide insufflation may lead deleterious changes in fetal carbon dioxide levels and acid/base status, ultimately affecting fetal well-being $[7,8]$. Currently two-dimensional echocardiography is used to monitor fetuses intraoperatively, [9] but this modality provides limited information on end-organ perfusion which, if compromised, could permanently affect organ development. Unfortunately, until now, there has been no reliable, noninvasive method to measure fetal brain perfusion in utero during fetal surgery.

Contrast-enhanced ultrasound (CEUS) is an emerging, minimally-invasive imaging technique that has been increasingly used to detect cerebral perfusion defects in neonates and adults [10-13]. Clinically, injection of gasfilled microbubbles allows enhanced visualization of blood vessels that rivals computed tomography without ionizing radiation and can be performed at the patient's bedside $[10,13,14]$. We hypothesized that CEUS could be used to monitor fetal cerebral perfusion during fetal surgery. To test our hypothesis, we studied catheterized mid-gestation fetal sheep ex utero and in utero under normal and PACI conditions to simulate fetal surgery. Our objectives were to determine: (1) could CEUS be used to monitor fetal cerebral perfusion during fetal surgery, and (2) does acid/base status or hemodynamics affect perfusion parameters.

\section{Methods}

All animals were treated according to protocols approved by the Institutional Animal Care and Use Committee at the Children's Hospital of Philadelphia. All animals were housed in an on-site animal facility. Ewes had consistent access to food and water and were allowed to feed ad libitum until the night prior to surgery.

\section{Anesthesia protocol}

Time-dated pregnant ewes were fasted and transdermal fentanyl patches $(2 \mu \mathrm{g} / \mathrm{kg} / \mathrm{hr})$ were applied to animal forelimbs 12 to $18 \mathrm{~h}$ prior to surgery. Ewes were selected when fetuses were gestational age 102-120 days which correlates to midgestation of a human fetus in terms of neurodevelopment [15]. Ewes were anesthetized with intramuscular ketamine $(15 \mathrm{mg} / \mathrm{kg})$ and inhaled isoflurane $\left(1-4 \%\right.$ in $\left.\mathrm{O}_{2}\right)$ and positioned in left lateral decubitus. Ventilator tidal volume $(8-10 \mathrm{ml} / \mathrm{kg} / \mathrm{min})$ and respiratory rate (10-18) were adjusted to achieve a maternal end-tidal $\mathrm{CO}_{2}$ of $35-40 \mathrm{~mm} \mathrm{Hg}$ and arterial $\mathrm{CO}_{2}$ of $35-45 \mathrm{~mm} \mathrm{Hg}$.

\section{Ex utero insufflation}

Six time-dated fetuses [113 \pm 5 days gestational age (GA)] were exteriorized via laparotomy and hysterotomy, cannulated via their umbilical vessels, and attached to a pumpless low resistance oxygenator circuit (Fig. 1 A). Fetal heart rate, arterial and venous blood pressure, umbilical blood flow, sweep gas flow rate and oxygen delivery were

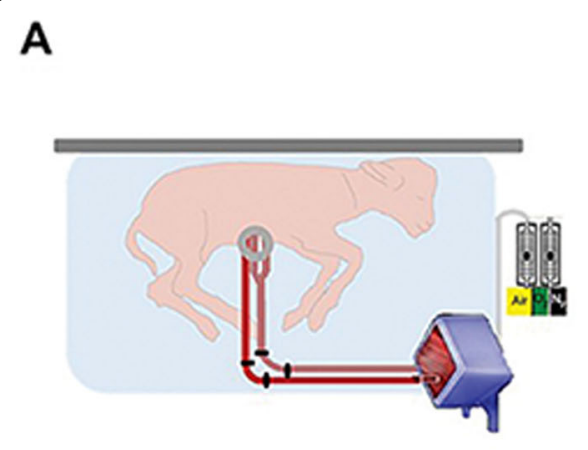

B
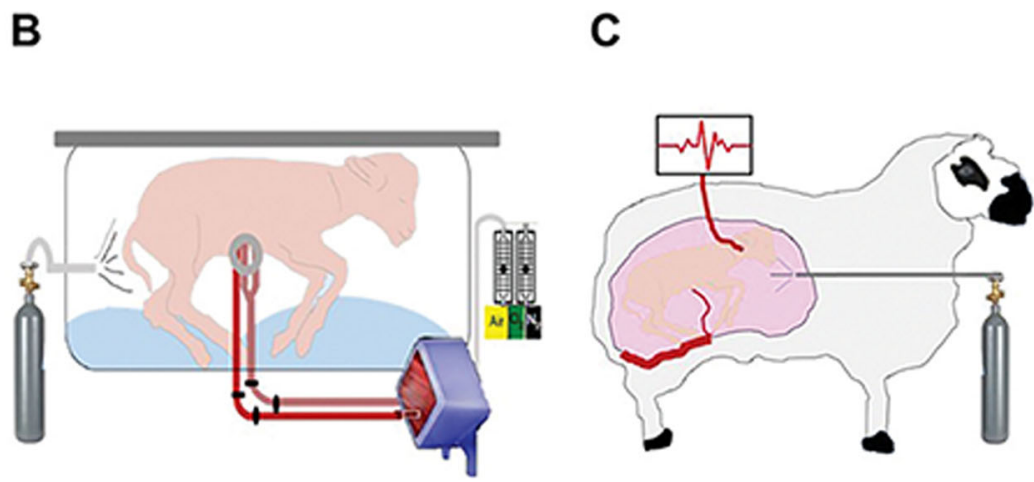

Fig. 1 Experimental conditions. a Fetal sheep $(n=6)$ were cannulated via their umbilical vessels, connected to a low resistance oxygenator circuit and supported in a fluid filled environment. $\mathbf{b}$ The fluid filled bag was partially drained and insufflated to 8 mm Hg with carbon dioxide to simulate fetal surgery.c The amniotic cavities of time-dated ewes $(n=4)$ were insufflated with carbon dioxide to simulate fetal surgery 
continuously measured (HT110 Bypass Meter and HXL Tubing Flowsensor Transonic Systems Inc., Ithaca, NY, USA) and recorded and (LabChart 5, ADInstruments Inc., Colorado Springs, CO, USA) as previously described [16]. During a 2-day acclimation period in this EXTrauterine Environment for Neonatal Development (EXTEND), physiologic levels of oxygen (15-20 $\mathrm{mmHg}$ ) and carbon dioxide (38-43 $\mathrm{mmHg}$ ) were maintained.

After two days, the sterile extra-uterine environment was partially drained of fluid and insufflated with $\mathrm{CO}_{2}$, helium or nitrogen to $8 \mathrm{mmHg}$ for $4 \mathrm{~h}$ (Karl Storz, Endoscopy Tuttlington, Germany) to mimic fetoscopic surgery conditions (Fig. 1B). Oxygenator sweep gas flow was held constant throughout the experimental period. Serial blood gases (i-Stat System, Abbott Point of Care Inc., Princeton, NJ, USA) were obtained and CEUS examinations were performed every $28 \pm 9$ min during insufflation as fetal positioned allowed. After a two-day recovery period and normalization of blood gas and hemodynamic variables, insufflation was repeated in these animals up to 5 occasions for a total of 20 experiments.

\section{In Utero Insufflation}

A mid-line laparotomy was performed, and the head of the fetus was exteriorized through a small hysterotomy at GA $103 \pm 2$. The fetal carotid artery was cannulated via neck incision with a polyvinyl catheter (ID 0.040", Scientific Commodities, Lake Havasu, AZ). The carotid catheter was exteriorized and secured to the ewe's flank. Prior to hysterotomy closure, amniotic fluid was replaced with warmed lactated ringers and treated with penicillin $\mathrm{G}$ (1 million units). The hysterotomy and laparotomy were closed, and ewes recovered from surgery.

After 2 days of recovery, baseline fetal arterial blood gas samples were obtained and ewes re-anesthetized. Fetuses GA $105 \pm 2$ days were evaluated in the operating room under maternal anesthesia with $(\mathrm{n}=4)$ or without $(\mathrm{n}=3)$ intra-amniotic $\mathrm{CO}_{2}$ insufflation to $8 \mathrm{mmHg}$ for $4 \mathrm{~h}$ with continuous hemodynamic assessment and serial blood gas analysis (Fig. $1 \mathrm{C}$ ). Throughout the surgical procedures, CEUS examinations were performed every $20 \mathrm{~min}$.

\section{Ultrasound evaluation}

CEUS examinations of the brain were performed by a board-certified radiologist throughout the ex utero and in utero studies. All imaging was performed with a C2$9 \mathrm{MHz}$ transducer on a commercially-available GE Loqiq E9 ultrasound system with contrast capabilities (GE Healthcare, Chicago, IL, USA).

Lumason $^{\circ}$ (sulfur hexafluoride lipid-type A microspheres; Bracco Diagnostics Inc., Monroe Township, NJ, USA) ultrasound contrast agent was administered as a bolus via the fetal umbilical vein (ex utero, $0.2 \mathrm{~mL}$ ) or via fetal arterial catheter (in utero, $0.3 \mathrm{~mL}$ ) with simultaneous ultrasound cine acquisition. Previous literature has documented CEUS safety data in the EXTEND model [17]. Ninety-second cine clips were obtained of the brain in a transaxial (ex utero) or coronal (in utero) plane which included the thalami (Figs. 2 and 3). CEUS examinations were analyzed (AS) using VueBox $^{\text {Txt }}$ (Bracco Imaging Suisse SA, Geneva, Switzerland).

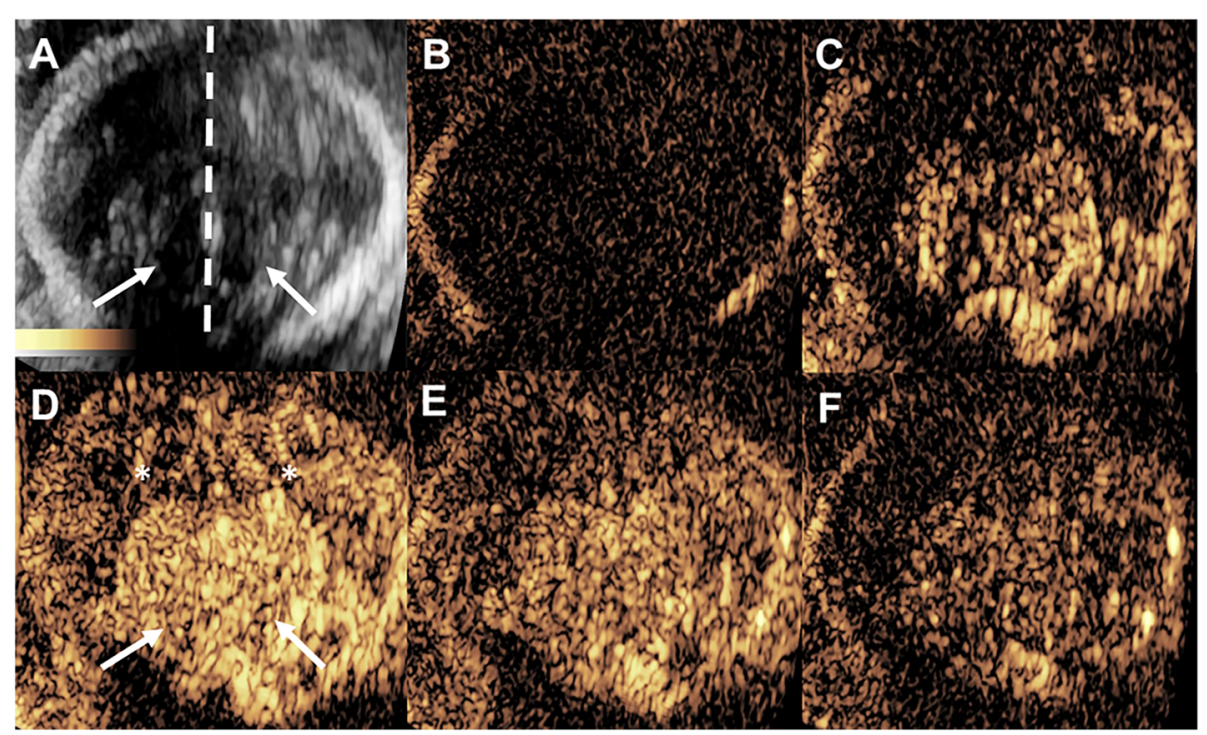

Fig. 2 Representative images from contrast-enhanced ultrasound examinations through the fetal brain in at various timepoints during ex utero transaxial imaging evaluation with a, grayscale image for localization including thalami (arrows), brain parenchyma (asterisks), and midline denoted by the dotted line and $\mathbf{b}$, baseline contrast optimized image. Following bolus ultrasound contrast administration, brain parenchymal perfusion is visualized at $\mathbf{c}$, early wash-in, $\mathbf{d}$, maximum enhancement of the thalami (arrows) and brain parenchyma (asterisks), e, early wash-out, and $\mathbf{f}$, late wash-out 


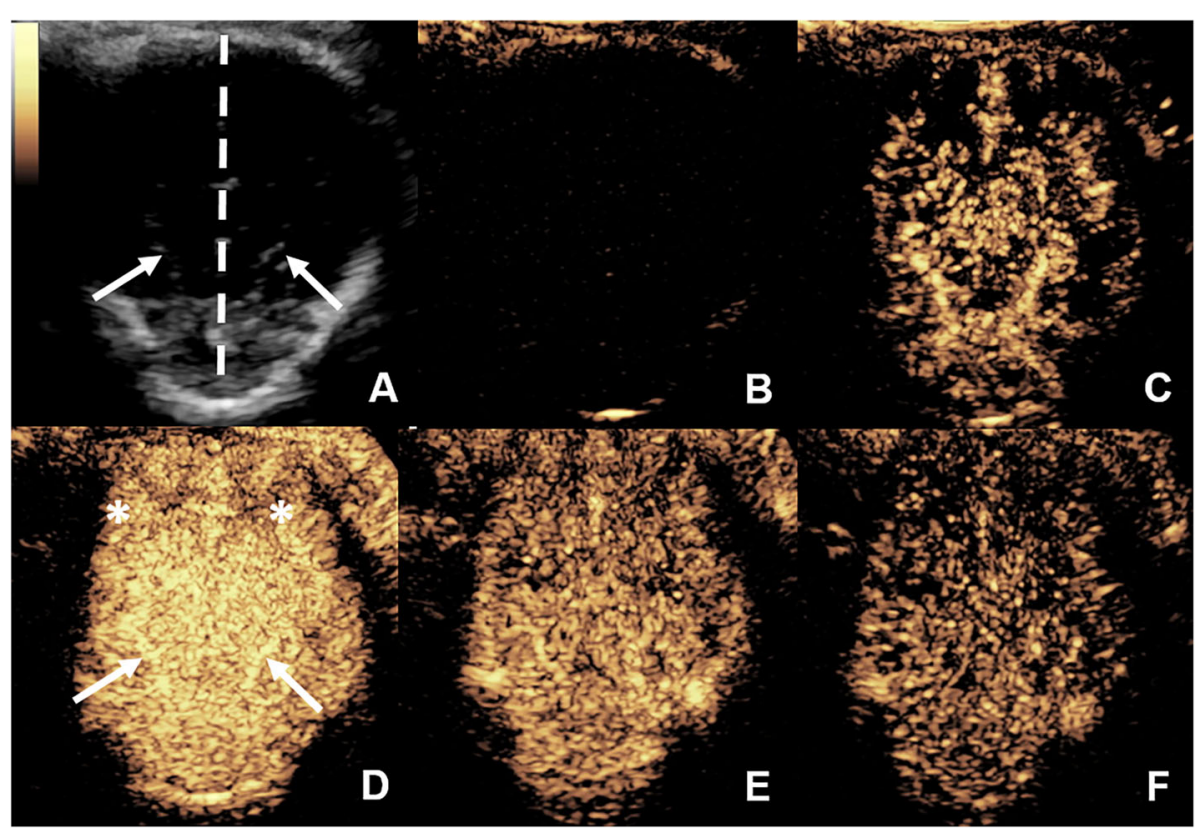

Fig. 3 In utero contrast-enhanced ultrasound examination through the fetal brain in a coronal plane with a, grayscale image for localization including thalami (arrows), brain parenchyma (asterisks), and midline denoted by the dotted line and $\mathbf{b}$, baseline image optimized for contrast visualization. Following bolus contrast administration, brain parenchymal perfusion is visualized at $\mathbf{c}$, early wash-in, $\mathbf{d}$, at maximum enhancement of the thalami (arrows) and brain parenchyma (asterisks), e, early wash-out, and $\mathbf{f}$, late wash-out

Regions-of-interest were placed to include the entire brain and time-intensity-curves were created. Perfusion metrics known to correlate with cerebral blood flow were obtained from the analyzed datasets including timeto-peak intensity (TTP), rise time (RT), fall time (FT), and transit time (TT). Due to the presence of recirculating ultrasound contrast material in this model, which artificially increases the softwaregenerated mean transit time, TT was defined as $\mathrm{RT}+\mathrm{FT}$ for the purposes of analysis $[10,13,18,19]$. CEUS examinations were excluded from analysis if quality of time-intensity-curve fit was $<80 \%$ with analyzed examinations represented in the flowchart (Fig. 4).

\section{Statistical analysis}

For extra-uterine experiments, hemodynamic parameters and arterial blood gas measurements were compared over time using mixed effects linear regression models with animal and study date analyzed as random effects. For in utero experiments, fetal hemodynamic parameters (heart rate and blood pressure) and arterial blood gas

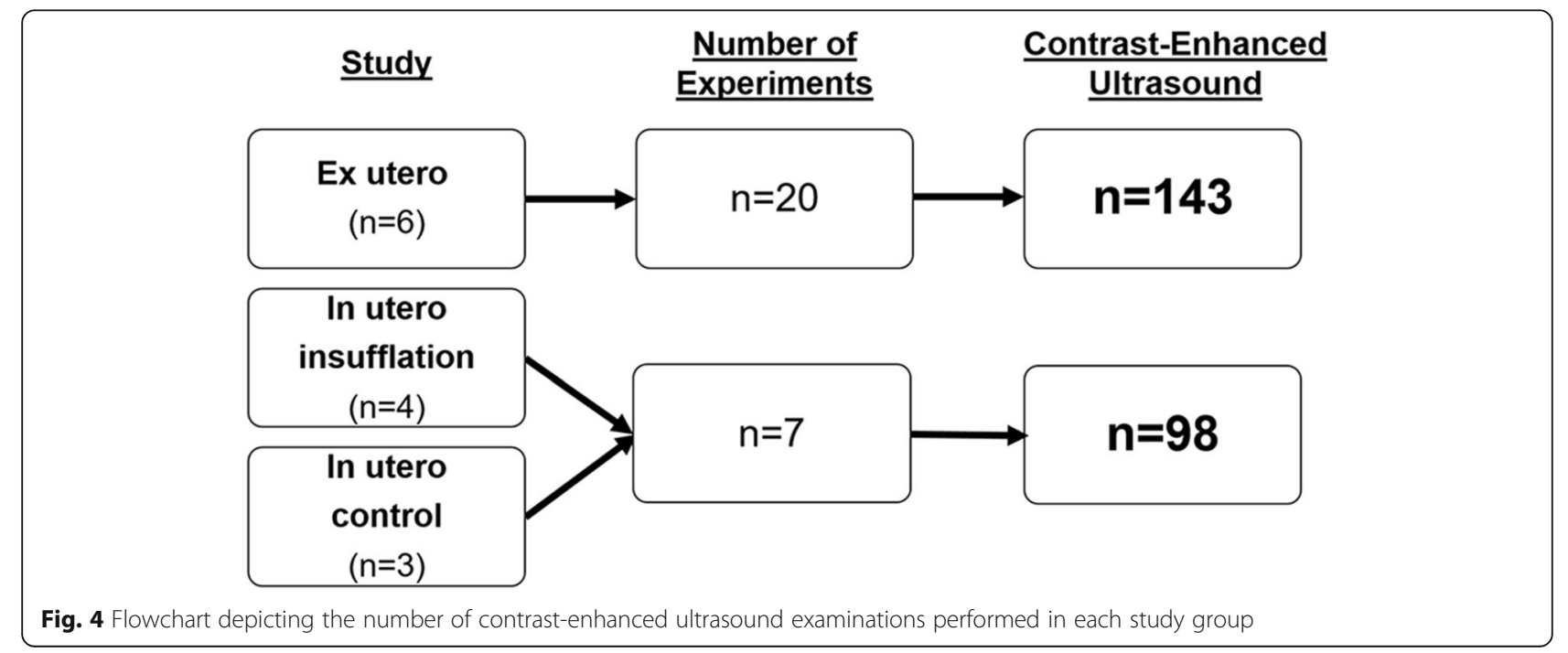


measurements for insufflated and control animals were compared over time using two-way repeated measure analysis of variance. Perfusion parameters obtained from ex utero and in utero studies were plotted over time and lines of best fit were obtained for each perfusion parameter. Mixed effects linear regression models were used to explore the relationships between perfusion variables and simultaneously obtained hemodynamic (heart rate, mean arterial pressure) and serial blood gas measurements. All data are presented as mean \pm standard deviation, unless otherwise stated. Significance was accepted at $p \leq 0.05$. GraphPad, version 5.0 (Prism, GraphPad Software, Inc. La Jolla, CA) and Stata, version 15 (Stata Statistical Software: Release 15, StataCorp LLC, College Station, TX) were used for statistical analysis and graphical depictions of data.

\section{Results}

\section{Ex utero insufflation}

Fetuses were insufflated for a total of 20 experiments within the EXTEND system for $4 \mathrm{~h}$ with one exception, one study was terminated early due to animal movement resulting in transient hemodynamic instability. The ultimate CEUS examination occurred at $218 \pm 37 \mathrm{~min}$ from insufflation initiation. Table 1 shows fetal hemodynamics and blood gas measurements. Throughout the study periods, fetal heart rate, mean arterial pressure and arterial oxygen content did not change significantly. In contrast, fetal $\mathrm{CO}_{2}$ increased over the study period from a baseline of $34 \pm 3$ to $43 \pm 11 \quad(p=<0.01)$ and $\mathrm{pH}$ declined from a baseline of $7.37 \pm 0.04$ to $7.24 \pm$ $0.08(p=<0.01)$.

Table 2 shows the relationship between CEUS perfusion parameters and measured fetal variables from mixed effect linear regression models. There is no significant relationship between any CEUS perfusion parameter and fetal oxygen content, bicarbonate level, heart rate or mean arterial pressure. There was a significant positive correlation between all perfusion parameters and $\mathrm{pH}$ and a significant negative correlation between all perfusion parameters and $\mathrm{pCO} 2$.

Table 1 Fetal variables ( $n=20$ experiments) over time in ex utero support device

\begin{tabular}{llll}
\hline & Baseline & Final & $P$ value \\
\hline $\mathrm{pH}$ & $7.37 \pm 0.04$ & $7.24 \pm 0.08$ & $<0.01^{*}$ \\
$\mathrm{pCO}_{2}(\mathrm{~mm} \mathrm{Hg})$ & $34 \pm 3$ & $43 \pm 11$ & $<0.01^{*}$ \\
$\mathrm{pO}_{2}(\mathrm{~mm} \mathrm{Hg})$ & $25 \pm 4$ & $27 \pm 5$ & 0.09 \\
$\mathrm{HCO}_{3}-(\mathrm{mEq} / \mathrm{L})$ & $19 \pm 3$ & $18 \pm 3$ & $0.01^{*}$ \\
Heart rate (beats per minute) & $171 \pm 15$ & $167 \pm 31$ & 0.08 \\
Mean arterial pressure $(\mathrm{mm} \mathrm{Hg})$ & $37 \pm 6$ & $39 \pm 5$ & 0.86 \\
\hline
\end{tabular}

Fetal variables were assessed over time with mixed effects linear regression models. Data shown as mean \pm standard deviation with statistically significant results denoted by *.
As an example of these relationships, post-estimation predictive margins following the mixed effects linear regression analyses were plotted for (Fig. $5 \mathrm{~A}$ ) transit time and $\mathrm{pCO} 2$ and (Fig. 5B) transit time and pH. Perfusion parameters decline with acidosis and hypercapnia despite stability of fetal hemodynamics and arterial oxygen content (Table 1).

\section{In Utero Insufflation}

Compared to non-insufflated fetuses, insufflated fetuses had significantly decreased $\mathrm{pH}$ levels and significantly increased arterial $\mathrm{CO}_{2}$ levels (Fig. 6 A-B). There was no difference in fetal arterial oxygen levels between noninsufflated and insufflated fetuses throughout the study period ( $21 \pm 3$ vs. $20 \pm 3 \mathrm{~mm} \mathrm{Hg}, p=0.76$ ) (Fig. $6 \mathrm{C}$ ). Fetal heart rate $(143 \pm 3$ vs. $145 \pm 13$ beats per minute, $p=0.84$ ) (Fig. $6 \mathrm{D})$ and mean arterial pressure $(40 \pm 6$ vs. $42 \pm 3 \mathrm{mmHg}, p=0.73)$ were also maintained with no difference between groups throughout the anesthesia period.

Insufflated fetuses had significantly reduced TTP ( $4.05 \pm 0.56$ vs. $7.73 \pm 1.26 \mathrm{~s}, p=0.03)$, RT ( $2.65 \pm 0.38$ vs. $5.24 \pm 0.58 \mathrm{~s}, p=0.01)$, FT $(5.34 \pm 0.79$ vs. $11.52 \pm 1.07 \mathrm{~s}$, $p<0.01)$ and TT $(7.99 \pm 1.17$ vs. $16.76 \pm 1.50 \mathrm{~s}, p<0.01)$ compared to non-insufflated fetuses (Fig. 6 A-B).

\section{Discussion}

In this study we utilized ex utero and in utero models to demonstrate that CEUS could be used to monitor fetal cerebral perfusion during fetal surgery. Furthermore, we found that disturbances in fetal acid/base balance correlated strongly with measured perfusion parameters. These findings have important implications for the field of fetal surgery, which is rapidly expanding, and associated monitoring techniques. In the future, this technology could be used for intra-operative assessment of fetuses to detect early acid/base or perfusion disturbances.

Prolonged insufflation led to fetal acidosis and hypercapnia without significant changes in hemodynamics or oxygenation. Prior in utero insufflation studies have similarly demonstrated severe acidosis in exposed fetal sheep $[5,7,8]$. Here we show that fetuses within EXTEND still develop hypercapnic acidosis, albeit much milder than in utero fetuses. The implications of this difference between our models on human fetuses undergoing fetoscopy is unclear, particularly as differences in placentation, uterine laxity, amniotic fluid, and/or skin keratinization in fetal sheep may limit direct translation of findings to humans and future studies are aimed to better delineate the mechanisms of fetal carbon dioxide absorption. However, currently, echocardiography is used to monitor fetal heart rate, systolic function, and valvular regurgitation intra-operatively, [9] but here we 
Table 2 Relationship between CEUS perfusion parameters and fetal variables ( $\mathrm{n}=20$ experiments)

\begin{tabular}{|c|c|c|c|c|c|c|c|c|c|c|c|c|}
\hline & \multicolumn{12}{|c|}{ Perfusion parameters (s) } \\
\hline & \multicolumn{3}{|c|}{ Time-to-peak intensity } & \multicolumn{3}{|l|}{ Rise time } & \multicolumn{3}{|l|}{ Fall time } & \multicolumn{3}{|l|}{ Transit time } \\
\hline & Coefficient & SE & $P$ value & Coefficient & SE & $P$ value & Coefficient & SE & $P$ value & Coefficient & SE & $P$ value \\
\hline $\mathrm{pH}$ & 7.09 & 3.18 & $0.03^{*}$ & 5.11 & 1.55 & $<0.01^{*}$ & 7.58 & 3.24 & $0.02^{*}$ & 12.35 & 4.11 & $<0.01^{*}$ \\
\hline $\mathrm{pCO}_{2}(\mathrm{~mm} \mathrm{Hg})$ & -0.12 & 0.04 & $<0.01^{*}$ & -0.74 & 0.02 & $<0.01^{*}$ & -0.09 & 0.03 & $0.01^{*}$ & -0.16 & 0.04 & $<0.01^{*}$ \\
\hline $\mathrm{pO}_{2}(\mathrm{~mm} \mathrm{Hg})$ & 0.02 & 0.07 & 0.72 & -0.00 & 0.03 & 0.96 & -0.03 & 0.07 & 0.7 & -0.03 & 0.09 & 0.7 \\
\hline $\mathrm{HCO}_{3}-(\mathrm{mEq} / \mathrm{L})$ & -0.05 & 0.11 & 0.67 & -0.01 & 0.05 & 0.82 & 0.03 & 0.16 & 0.86 & 0.00 & 0.19 & 0.99 \\
\hline Heart rate (beats per minute) & 0.01 & 0.01 & 0.39 & 0.00 & 0.00 & 0.94 & -0.02 & 0.00 & 0.07 & -0.02 & 0.01 & 0.15 \\
\hline Mean arterial pressure $(\mathrm{mm} \mathrm{Hg})$ & 0.07 & 0.05 & 0.11 & 0.00 & 0.02 & 0.72 & 0.02 & 0.05 & 0.69 & 0.03 & 0.06 & 0.67 \\
\hline
\end{tabular}

Relationship between perfusion parameters and fetal variables determined with mixed linear effects models presented as coefficient, standard error (SE), and $p$ value with statistically significant results denoted by *.

showed that metabolic and brain perfusion disturbances can occur without changes in heart rate or blood pressure implying that echocardiography has limited utility in detecting these early disturbances and improved methods of detecting aberrations in fetal physiology are warranted.

Hypercapnia and acidosis cause cerebral vasodilation which results in increased cerebral blood flow [20]. Increased cerebral blood flow is particularly poorly tolerated in fetuses, because of impaired cerebral autoregulatory mechanisms and the presence of fragile germinal matrix blood vessels [21, 22]. Therefore, early detection of acid/base or perfusion derangements is critical to prevent neurologic sequelae [21-23]. Our data demonstrate that CEUS can be used to measure cerebral perfusion during simulated fetal surgery. Importantly, we also showed that the measured time-dependent perfusion parameters including RT, FT, and TT, all decreased with hypercapnia and acidosis indicating faster/increased cerebral blood flow, assuming a constant fetal blood volume $[10,11]$. In other words, CEUS detected increased cerebral blood flow when fetuses became acidotic or hypercapnic during fetal surgery making this a potentially useful monitoring tool during fetal surgery.

In contrast to $\mathrm{pH}$ and $\mathrm{pCO}_{2}$, measured cerebral perfusion parameters did not change with fetal heart rate or blood pressure. Maintenance of cerebral blood flow under these conditions suggests that lamb fetuses at this gestational age can autoregulate cerebral blood flow to a certain extent. These results, however, may underestimate the effects of fetal hemodynamics on cerebral blood flow. First, we did not evaluate volume-dependent perfusion variables such as wash-in and wash-out area under curve which may be more sensitive to changes in hemodynamics [13]. Second there was no significant change in fetal heart rate or blood pressure throughout the studies which limited our ability to study associated perfusion changes. Future studies are planned to analyze both time and volume-dependent perfusion variables over a range of hemodynamic parameters to better understand fetal autoregulation of cerebral blood flow during surgery.
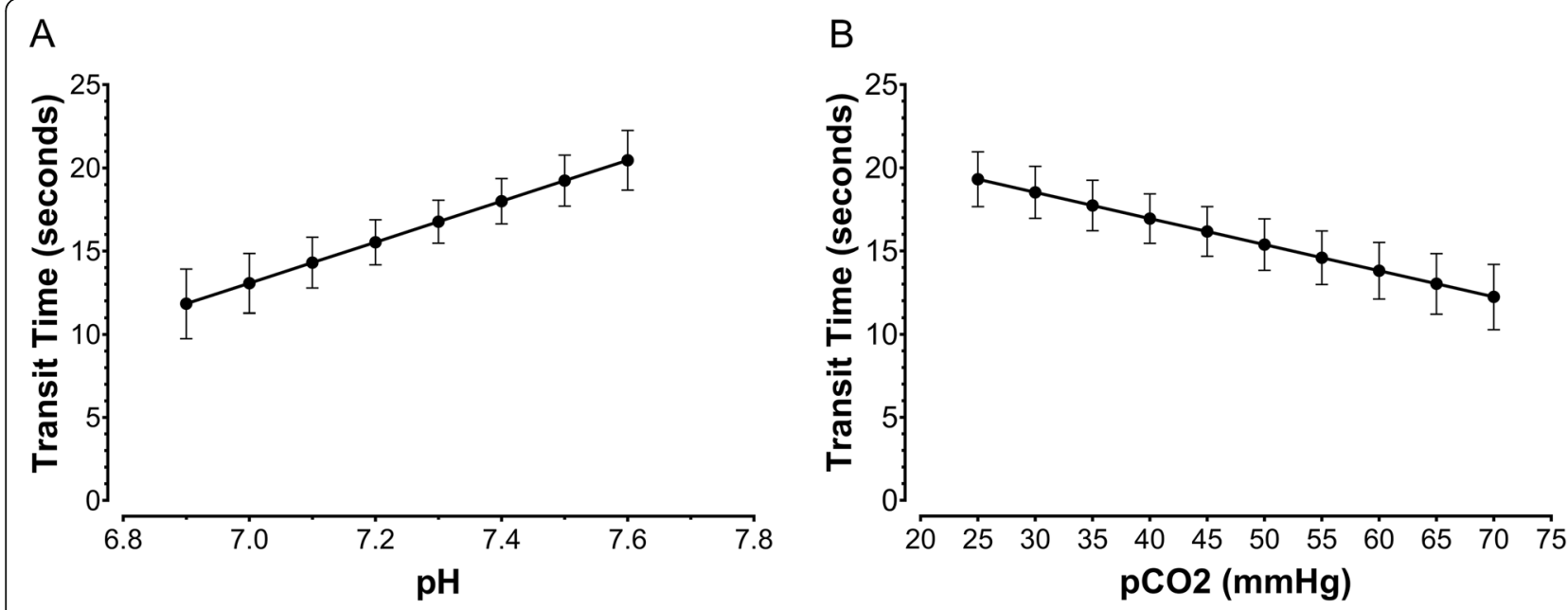

Fig. 5 Relationship between representative brain perfusion parameters and serum laboratory values ( $n=20$ experiments). Predictive margins following mixed effects linear regression analyses of brain perfusion parameters and serum laboratory values from ex utero contrast-enhanced ultrasound examinations were plotted for $\mathbf{a}$, transit time and $\mathrm{pCO} 2$ and $\mathbf{b}$, transit time and pH. Error bars show the standard error 


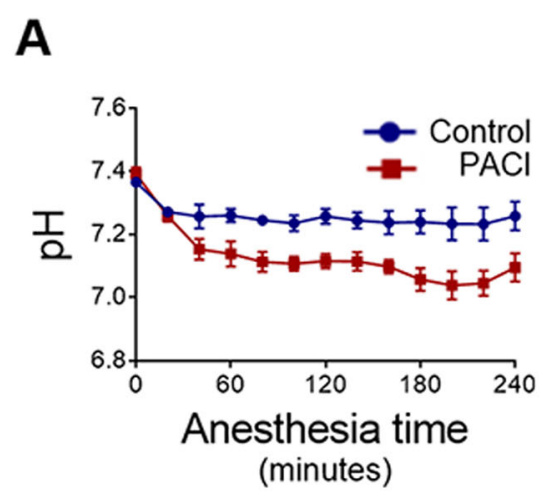

C

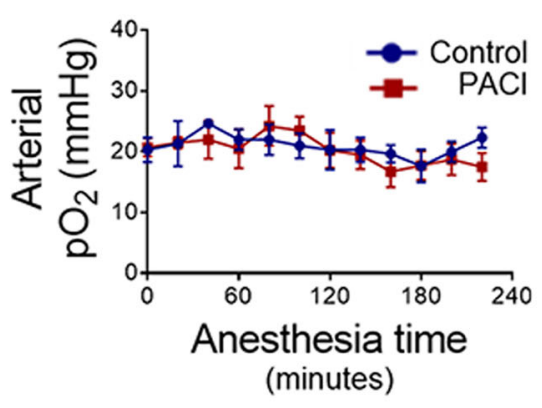

B

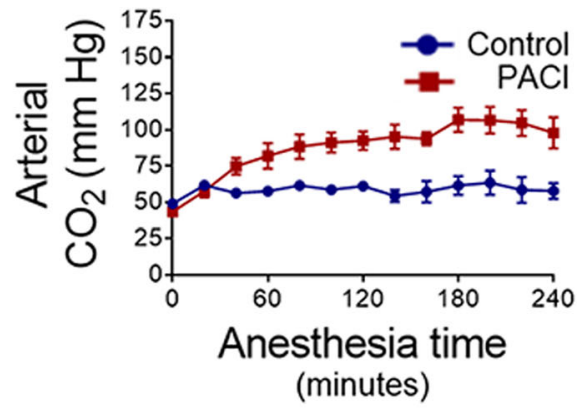

D

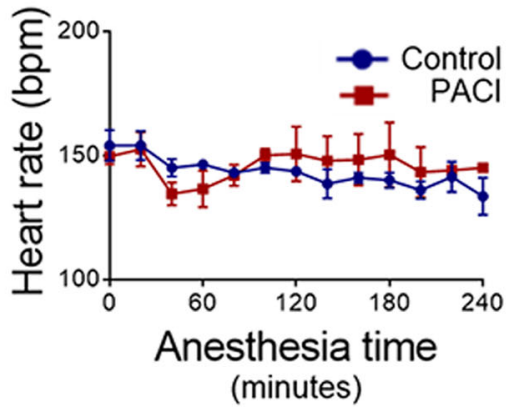

Fig. 6 Evaluation of serum laboratory values and fetal heart rate in non-insufflated control fetuses $(n=3)$ and insufflated fetuses ( $n=4)$ over time. In uteroa, pH and $\mathbf{b}$, arterial carbon dioxide levels increased significantly in insufflated fetuses versus non-insufflated control fetuses. $\mathbf{c}$ Oxygen levels and $\mathbf{d}$, heart rate were unchanged in insufflated fetuses versus non-insufflated control fetuses. Data shown as mean \pm standard deviation

\section{Limitations}

There were several additional limitations to this study which may limit clinical utility. First, contrast was delivered via umbilical vein or carotid artery which would not be feasible using current surgical methods during minimally invasive fetoscopic procedures. Prior to clinical use, trans-placental delivery of ultrasound contrast agents must be refined and is an area of ongoing research. Second, normative fetal cerebral perfusion data does not exist, so measured changes in cerebral perfusion may be difficult to interpret in early implementation of this technique. Future studies will be aimed to establish normative data and perform subsequent MRI to better understand neurologic implications of perfusion changes. Next, while we were able to demonstrate significant relationships, sample size was limited for these studies which increases the likelihood of a type two error. Finally, perfusion parameters are calculated by interpretation of contrast kinetics, and thus, is an intermittent monitoring technique. Ideally, fetal cerebral perfusion would be measured continuously during fetal surgery, and future exploration into refining contrast kinetic analyses to minimize gaps in fetal monitoring would be useful before intra-operative implementation.

\section{Conclusions}

CEUS can be used to measure cerebral perfusion during simulated fetal surgery in sheep. Importantly, we also found that measured perfusion parameters correlate with hypercapnia and acidosis which occurred during prolonged insufflation. With further development, CEUS could be a useful tool for intra-operative fetal monitoring during investigative or clinical fetal surgery.

\section{Abbreviations}

CEUS: Contrast-enhanced ultrasound; EXTEND: EXtra-uterine Environment for Neonatal Development; FT: Fall time; GA: Gestational age; PACl: Partial Amniotic Carbon Dioxide Insufflation; RT: Rise time; TT: Transit time; TTP: Time-To-Peak intensity

\section{Acknowledgements}

We gratefully acknowledge Elizabeth Rowley, MS for statistical support and analysis and Grace Hwang, BA, Kathleen Young, BS, and Aaron Weilerstein BS for their technical assistance. We thank the Department of Radiology at the Children's Hospital of Philadelphia for their equipment and personnel support. Data included in this manuscript were presented in oral format at the $49^{\text {th }}$ Annual American Pediatric Surgery Association meeting in Palm Desert, California on May 3 to 6, 2018, the European Society of Pediatric Radiology meeting in Helsinki, Finland on May 14-15, 2019, and the International Fetal Medicine and Surgery Society meeting in Sils, Switzerland on October 22 to 26, 2019. 


\section{Authors' contributions}

KML contributed to the study design, data acquisition, analysis, and interpretation, and manuscript drafting and revision. BEC contributed to the study design, data acquisition, analysis, and interpretation, and manuscript revision. AS contributed to data analysis, interpretation of data, and manuscript drafting and revision. ACR contributed to study design and data acquisition. MGD contributed to study conception and design. AWF contributed to study conception and design. RAD contributed to study design, data acquisition, analysis, and interpretation, and manuscript drafting and revision. All authors read and approved the final manuscript.

\section{Funding}

Research reported in this publication was supported by the Radiologic Society of North America Research Scholar Program and the National Center for Advancing Translational Sciences of the National Institutes of Health under Award Number UL1TR001878 and TL1TR001880. The content is solely the responsibility of the authors and does not necessarily represent the official views of the NIH. Supported in part by the Institute for Translational Medicine and Therapeutics (ITMAT) Transdisciplinary Program in Translational Medicine and Therapeutics.

\section{Availability of data and materials}

The data generated and analyzed during the current study are available from the corresponding author on reasonable request.

\section{Declarations}

\section{Ethics approval}

All animals were treated according to protocols approved by the Institutional Animal Care and Use Committee at the Children's Hospital of Philadelphia.

\section{Consent for publication}

Not applicable.

\section{Competing interests}

The authors report no financial conflict of interest. MGD and AWF are patent holders on the described extra-uterine support device.

\section{Author details}

'Center for Fetal Research, Department of Surgery, The Children's Hospital of Philadelphia Research Institute, 3401 Civic Center Blvd, Pennsylvania 19104 Philadelphia, USA. 'Department of Radiology, The Children's Hospital of Philadelphia, 3401 Civic Center Blvd.. PA PA 19104 Philadelphia, USA ${ }^{3}$ Perelman School of Medicine, University of Pennsylvania, Pennsylvania, Philadelphia, USA.

Received: 18 November 2020 Accepted: 1 March 2021

Published online: 30 March 2021

\section{References}

1. Kett JC, Woodrum DE, Diekema DS. A survey of fetal care centers in the United States. J Neonatal Perinatal Med. 2014;7(2):131-5.

2. Moon-Grady AJ, Baschat A, Cass D, et al. Fetal Treatment 2017: The evolution of fetal therapy centers - A joint opinion from the International Fetal Medicine and Surgical Society (IFMSS) and the North American Fetal Therapy Network (NAFTNet). Fetal Diag Ther. 2017;42(4):241-8.

3. Pedreira DA, Zanon N, Nishikuni K, et al. Endoscopic surgery for the antenatal treatment of myelomeningocele: the CECAM trial. Am J Obstet Gynecol. 2014;214(1):111.e111.

4. Ziemann M, Fimmers R, Khaleeva A, et al. Partial amniotic carbon dioxide insufflation (PACl) during minimally invasive fetoscopic interventions on fetuses with spina bifida aperta. Surg Endosc. 2018:32(7):3138-48.

5. Belfort MA, Whitehead WE, Shamshirsaz AA, et al. Fetoscopic open neural tube defect repair: Development and refinement of a two-port, carbon dioxide insufflation technique. Obstet Gynecol. 2017;129(4):734-43.

6. Adzick NS, Thom EA, Spong CY, et al. A randomized trial of prenatal versus postnatal repair of myelomeningocele. N Engl J Med. 2011;364(11):9931004.

7. Luks Fl, Deprest J, Marcus M, et al. Carbon dioxide pneumoamnios causes acidosis in fetal lamb. Fetal Diagn Ther. 1994;92(2):105-9.
8. Gratacos E, Wu J, Devlieger R, Van de Velde M, et al. Effects of amniodistention with carbon dioxide on fetal acid-base status during fetoscopic surgery in a sheep model. Surg Endosc. 2001;15(4):368-72.

9. Rychik J, Cohen D, Tran KM, et al. The role of echocardiography in the intraoperative management of the fetus undergoing myelomeningocele repair. Fetal Diagn Ther. 2015;37(3):172-8.

10. Hwang M, Sridharan A, Darge K, et al. Novel quantitative contrast-enhanced ultrasound detection of hypoxic ischemic injury in neonates and infants: pilot study 1. J Ultrasound Med. 2019;38(8):2025-38.

11. Hwang M, De Jong RM, Herman S, et al. Novel contrast-enhanced ultrasound evaluation in neonatal hypoxic ischemic injury: Clinical application and future directions. J Ultrasound Med. 2017;36(11):2379-86.

12. Seidel G, Meairs S. Ultrasound contrast agents in ischemic stroke. Cerebrovasc Dis. 2009;27(Supple 2):25-39.

13. Vinke EJ, Kortenbout AJ, Eyding J, et al. Potential of contrast-enhanced ultrasound as a bedside monitoring technique in cerebral perfusion: a systematic review. Ultrasound Med Biol. 2017:43(12):2751-7.

14. Meairs S, Kern R. Intracranial perfusion imaging with ultrasound. Front Neurol Neurosci. 2015;36:57-70.

15. Back SA, Riddle A, Hohimer AR. Role of instrumented fetal sheep preparations in defining the pathogenesis of human periventricular whitematter injury. J Child Neurol. 2006:21(7):582-9.

16. Partridge EA, Davey MG, Hornick MA, et al. An extra-uterine system to physiologically support the extreme premature lamb. Nat Commun. 2017;8: 15112.

17. Didier RA, Sridharan A, Lawrence K, Coleman BG, Davey MG, Flake AW. Contrast-enhanced ultrasound in extracorporeal support: In vitro studies and initial experience and safety data in the extreme premature fetal lamb maintained by the extrauterine environment for neonatal development. J Ultrasound Med. 2019;38(8):1971-8.

18. Kastelr A, Manzoni P, Chapuy S, et al. Transfontanellar contrast enhanced ultrasound in infants: Initial experience. J Neuroradiol. 2014;41(4):251-8.

19. De Lange C, Brabrand K, Emblem KE, et al. Cerebral perfusion in perinatal hypoxia and resuscitation assessed by transcranial contrast-enhanced ultrasound and 3 T MRI in newborn pigs. Invest Radiol. 2011:46(11):686-96.

20. Levene MI, Fawer CL, Lamont RF. Risk factors in the development of intraventricular haemorrhage in the preterm neonate. Arch Dis Child. 1982: 57(6):410-7.

21. Kaiser JR, Gauss CH, Williams DK. The effects of hypercapnia on cerebral autoregulation in ventilated very low birth weight infants. Pediatr Res. 2005; 58(5):931-5.

22. Perlman JM, Mcmenamin JB, Volpe JJ. Fluctuating cerebral blood-flow velocity in respiratory-distress syndrome: Relation to the development of intraventricular hemorrhage. N Engl J Med. 1983:309(4):204-9.

23. Kaiser JR, Gauss $\mathrm{CH}$, Pont MM, et al. Hypercapnia during the first 3 days of life is associated with severe intraventricular hemorrhage in very low birth weight infants. J Perinatol. 2006;26(5):279-85.

\section{Publisher's Note}

Springer Nature remains neutral with regard to jurisdictional claims in published maps and institutional affiliations.

Ready to submit your research? Choose BMC and benefit from:

- fast, convenient online submission

- thorough peer review by experienced researchers in your field

- rapid publication on acceptance

- support for research data, including large and complex data types

- gold Open Access which fosters wider collaboration and increased citations

- maximum visibility for your research: over $100 \mathrm{M}$ website views per year

At BMC, research is always in progress.

Learn more biomedcentral.com/submissions 